\title{
Seis epígrafes para la disciplina escolar: política educativa chilena reciente
}

\author{
María Angélica Oliva
}

\section{Resumen:}

El artículo analiza la disciplina escolar en su correspondencia con el orden social y su expresión en la política educativa chilena, en especial, en las Bases Curriculares Nacionales vigentes. Se argumenta que la disciplina constituye el dispositivo par excellence del orden escolar, por su vocación normalizadora de sujetos, saberes y discursos; en el caso de las Bases Curriculares Nacionales permite observar la estandarización del conocimiento escolar, en un currículum técnico que es funcional al orden neoliberal. La investigación documental, unida a una técnica hermenéutica, pone en evidencia la relevancia de la disciplina en la institución educativa al regular sujetos, saberes y discursos; en las Bases Curriculares Nacionales el objetivo de aprendizaje es el dispositivo disciplinario que renueva un currículum técnico, inaugurado con la pedagogía por objetivos en la reforma educacional de 1965, profundizado en la dictadura, y perfeccionado en los Gobiernos de la Concertación, pese a su funcionalidad con la reproducción social y su contribución a generar desigualdad. La magnitud de la desigualdad educativa en Chile requiere una Política de la Reparación, para impulsar una transmutación ética y política desde el círculo vicioso de la disciplina a su espiral virtuosa.

Palabras clave:

Chile; política educativa chilena; política de la reparación; disciplina escolar; conocimiento escolar. 


\title{
Seis epígrafes para a disciplina escolar: política educacional recente do Chile
}

Resumo: $O$ artigo discute a disciplina escolar em suas relações com a ordem social como expressão da política educacional chilena, principalmente nas diretrizes curriculares atuais. Argumenta-se que a disciplina, por excelência, represente o dispositivo de ordem escolar por sua vocação à normalização dos sujeitos e conhecimentos. Especificamente no caso das diretrizes curriculares, pode-se observar a padronização do conhecimento escolar em um currículo técnico que é funcional para a ordem neoliberal. A pesquisa documental valendo-se da hermenêutica como técnica, demonstra a importância da disciplina na escola ao regular conhecimento e discursos. A pesquisa ratifica a importância da disciplina na escola ao regular conhecimento e discursos. Nas diretrizes curriculares, o objetivo do aprendizado é o mecanismo disciplinar que renova um currículo técnico que teve início com a reforma educacional de 1965. O período da ditadura teria aprofundado e se aperfeiçoado nos Governos da Concertación, apesar de sua funcionalidade com a reprodução social e sua contribuição para a geração de desigualdade. A dimensão da desigualdade educacional no Chile requer uma Politica da Reparação para promover uma transmutação ética e política desde um círculo vicioso de disciplina para sua espiral virtuosa.

Palavras-chave: Chile; política educacional chilena; política da reparação; disciplina escolar; conhecimento escolar.

\section{Six épigraphes pour la discipline scolaire: la politique éducative récente du Chili}

Résumé: Cet article analyse la relation entre la discipline scolaire et l'ordre social, à travers son expression dans la politique éducative du Chili, en particulier, dans les programmes scolaires. Notre hypothèse est que la discipline est le dispositif par excellence de l'ordre scolaire par sa vocation de normalisation des sujets et des connaissances. Dans le cas des programmes scolaires, on observe la standardisation des savoirs à travers des programmes techniques ad hoc de l'ordre néolibéral. La recherche documentaire, à l'aide d'une technique herméneutique, permet de mettre en évidence l'importance de la discipline dans l'institution scolaire. La discipline normalise les gens, les connaissances et les discours. Dans les programmes scolaires, l'objectif d'apprentissage constitue le mécanisme disciplinaire qui avance un programme technique initié par la Pédagogie par Objectifs lors de la réforme éducative de 1965. Ce mécanisme a été par la suite approfondi pendant la dictature et perfectionné par les gouvernements de la Concertation, et cela, en dépit de sa contribution à la reproduction sociale et sa contribution à la production d'inégalités. L'ampleur des inégalités scolaires au Chili nécessite la mise en œuvre d'une " politique de la réparation " afin de promouvoir la transformation éthique et politique du cercle vicieux de la discipline vers sa spirale vertueuse.

Mots-clés: Chile; politique éducative chilienne; " politique de la réparation "; discipline scolaire; connaissances scolaires.

\section{Six epigraphs for the school discipline in Chile's recent educational policy}

\begin{abstract}
The article analyzes the school discipline aligned with the social order and its expression in the Chilean educational policy, particularly under the current curriculum guidelines. The hypothesis is that the school's discipline is par excellence a device that reproduces the school order due to its normalizing vocation and knowledge dissemination. The curriculum allows to observe the normalization of the school knowledge through a technical curriculum that it is functional to the neoliberal order. Documentary research as well as the use of a hermeneutic approach highlights the importance of the school discipline in regards to the normalization of individuals, as well as the educational institution's subjects, knowledge, and discourse. Despite the functionality to the social reproduction its contribution to generate inequality, the learning objective of the curriculum is the disciplinary mechanism that renews the technical one which was initiated with the pedagogy by objectives in the educational reform of 1965 , deepened in the dictatorship and perfected in the democratic governments (1990-2015). Finally, the extent of educational inequality in Chile requires a policy of reparation or, in other words, one that promotes ethical and political transmutation that moves from a vicious cycle of discipline to a virtuous spiral.
\end{abstract}

Keywords: Chile; Chilean educational policy; policy of reparation; school discipline; school knowledge. 


\section{Se Podes Olhar, Vê. Se Podes Ver, Repara} José Saramago

\section{Primer epígrafe: la disciplina en el lugar de la interrogación. Introito}

Memoria vestida de escuela es el lugar de la interrogación que anima a este texto. La Escuela de Niñas N¹7, emplazada en Recoleta, Santiago de Chile, en la antesala de la promulgación de la Ley de Instrucción Primaria, Obligatoria y Común, que regula la obligación escolar¹.

El despertar del siglo pasado permite inquirir: ¿Por qué maestra, alumnas y conocimiento escolar -en el globo terráqueo- giradas en $180^{\circ}$ mantienen, ordenadamente, su posición? (Fig.1). ¿De qué tipo es ese orden cuya subversión, paradojalmente, descubre el círculo de disciplinarización de sujetos y saberes propio de lo escolar? La metáfora iconográfica es el dispositivo que sitúa la interrogación en su contexto; obsérvese los signos de la disciplina en cuerpos, gestos y su distribución espacial, también, en el globo terráqueo, síntesis de la materia de enseñanza en un cosmos, funcional y funcionario de una cosmovisión que enseña la correspondencia entre orden escolar y orden social.

Ayer y hoy son los confines de la interrogación; el estudio del lugar de la memoria permite descubrir procesos de larga duración, como la disciplina, sobre los cuales ocurren determinados cambios. ¿Cuál es la naturaleza de la disciplina escolar que expresa su correspondencia -traducción, producción y reproducción- con el orden social? El artículo asume el reto de la hipótesis como el acto creativo par excellence de la razón indagadora: la disciplina en el cumplimiento de su vocación normalizadora de saberes y sujetos constituye el mecanismo y la máquina de lo escolar; un dispositivo para la invención del orden escolar y social, en fin, una matriz que ordena lo escolar.

Al tenor de la racionalidad técnica engastada en nuestra política educativa, puede pensarse en la capacidad instituyente que alienta la Reparación. Por ello, el estudio de la disciplina posee un sentido político, refiere al mundo en común, al espacio público y a la democracia que, precisamente, la disciplina puede fortalecer o debilitar. Luego, es imperativo trabajar en la dimensión de lo posible, desde la utopía para intentar fisurar el círculo vicioso creado por la disciplinarización de los saberes y sujetos (Varela, 1995); raison d'être de toda esta palabra.

Seis epígrafes para la disciplina escolar indagan en la disciplina, se focaliza el análisis en la disciplinarización de los saberes para escrutar el estatuto de la disciplina. Tras este primer epígrafe, que sitúa a la disciplina en el lugar de la interrogación; el segundo epígrafe, considera la ambivalencia de la voz disciplina para reflexionar en la disciplina necesaria para la comprensión del orden escolar; el tercer epígrafe, 
analiza la disciplinarización del conocimiento escolar y su proceso de normalización; la radicalización de lo disciplinario es el leitmotiv del cuarto epígrafe, que indaga en el conocimiento escolar estandarizado en las Bases Curriculares Nacionales; el quinto epígrafe aborda la encrucijada de la disciplina escolar e inquiere por las posibilidades y límitaciones de la educación. Exhortación a una Pedagogía de la Reparación, es la cristalización de este trabajo en el sexto epígrafe; reparar es darse cuenta y también, es actuar en consecuencia con la espiral virtuosa de la disciplina erigida en la mejor tradición pedagógica, que considera el acervo de la educación democrática y el derecho a la educación.

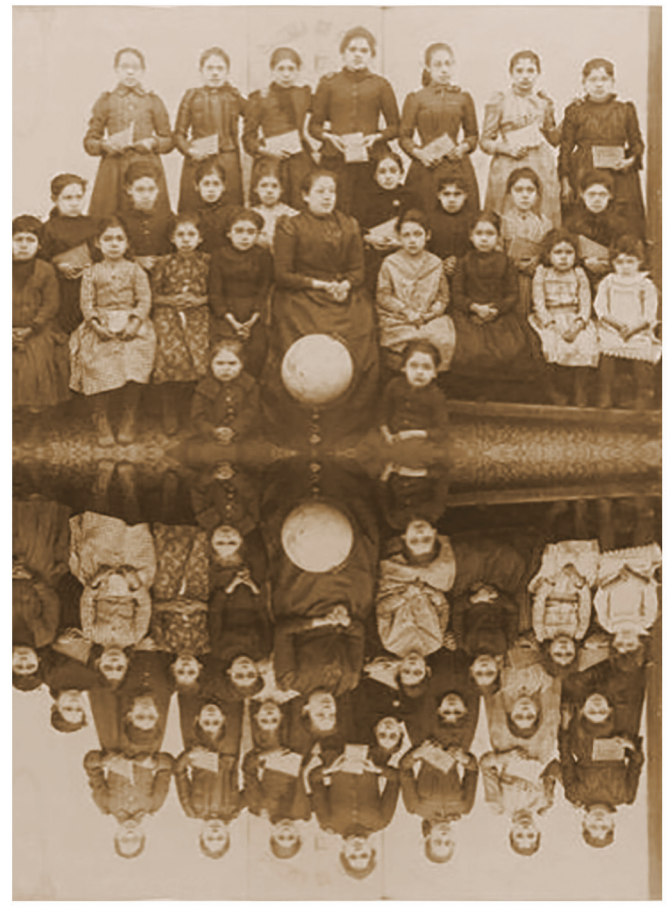

Fig. 1. Disciplina in péctore. Colección fotográfica Museo de la Educación Gabriela Mistral, Chile

Seis Epígrafes Antiguos de Claude Debussy inspiran este artículo; su piano sonoro y colorido rememora impression, soleil levant y alienta, a su paso, a cada epígrafe a profundizar en la comprensión de la disciplina escolar. Se trata, como en la epigrafía, de una tarea de desciframiento en diferentes estratos de la disciplina, cuya génesis es el orden social moderno del siglo XVIII, ligado al establecimiento de la sociedad disciplinaria (Foucault, 2014); la actual sociedad del control expresa un nuevo estrato de la disciplina (Oliva, 2012). Pour remercier la pluie du matin, sexto epígrafe debussiano, 
es un mensaje de arrojo para sellar un compromiso político, urgente en Chile por la magnitud de la inequidad que ha de repararse, pues, "La productividad y la desigualdad también se ven afectadas por el sistema educativo, cuyos resultados son débiles y desiguales, y reflejan en gran medida los antecedentes socioeconómicos de los alumnos" (Organisation for Economic Cooperation and Development [OECD], 2018, p.7).

\section{Segundo epígrafe: disciplina para la comprensión del orden escolar}

El epígrafe indaga en el orden escolar situándose en la anfibología de la voz disciplina: disciplina para disponer el ánimo a las exigencias del proceso de conocer y disciplina para descifrar el orden escolar. "Una vez más, la cuestión de la disciplina", escribe Paulo Freire (2006, p.128), alentando toda esta palabra. ¿Qué disciplina intelectual se requiere para estudiar la disciplina escolar? Parece posible pensar la disciplina como una matriz; una entidad principal que contiene y engendra a otras, imaginar un útero o la disposición ordenada de un conjunto de elementos (Larousse, 2014). Tal como la arquitectura de la política educativa chilena instituida en el dispositivo matriz de la subsidiariedad descubre, en la disciplina, un mecanismo y una máquina para la conformación del orden escolar. Uno de cuyos dispositivos es el currículum técnico, articulado en la medición. En este orden de cosas, la idea de dispositivo ita cara a Foucault, es un poderoso instrumento para descifrar lo escolar atendiendo a los discursos que se implementan y generan; al saber en sus curvas de visibilidad y enunciación en sus formaciones históricas; a las relaciones de fuerza (poder) que atraviesan todo lo anterior; y, finalmente, a las líneas de subjetivación, pliegues o la interioridad del pensamiento (Foucault, 2014; Agamben, 2011).

¿A qué se convoca al orden y a la disciplina? Cosmos y orden social permiten esclarecer, desde el macrocosmos, este interrogante que recala en el microcosmos de lo escolar. En nuestra civilización, la idea de orden detenta la condición de una matriz, la filosofía griega sostiene que el mundo aparece como algo ordenado y sometido a ley: esta es la noción de cosmos (Marías, 1971). En el dominio de lo humano, su legalidad configura el orden social, orden institucional o normativo que regula la acción humana mediante dos formas de disciplina; el sistema normativo, fundado en normas explícitas, y el sistema de normalidad, fundado en normas implícitas, cuna del poder simbólico (González Ramella, 2003). ¿A qué se convoca, entonces, al orden escolar y a su disciplina? Se trata de una función normalizadora, que deviene en una vocación disciplinadora en el umbral de la modernidad, con un conjunto de procesos que contribuyen a forjar una racionalidad disciplinaria, tales como: el surgimiento del Estado y la sociedad moderna, la llustración, las reformas religiosas, el desenvolvimiento del capitalismo industrial y la cristalización del orden científico, entre otros (Foucault, 2014). En esa racionalidad, no obstante la durée de la disciplina, la novedad impregna su sentido 
disciplinario, v.gr., en el Estado moderno la mudanza del modelo de soberanía, con sus técnicas disciplinarias fundadas en la fuerza, al modelo de dominación, con sus técnicas disciplinarias fundadas en el control, la coerción y la sujeción del cuerpo. Forman parte de esa racionalidad, las técnicas para el ordenamiento de la multiplicidad que persiguen maximizar la economía en el ejercicio del poder, los efectos de ese poder y su utilidad. Lugar donde la disciplina muestra el acoplamiento del poder de la norma a otros poderes, tales como: la ley, la palabra, el texto y la tradición. De especial interés para este trabajo es el examen, instrumento del poder disciplinario, síntesis de otros medios de buen encauzamiento, vigilancia jerarquizada y sanción normalizadora que cumple la función de calificar, clasificar y castigar yuxtaponiendo relaciones de poder y saber (Foucault, 2014).

Intitulada Disciplina in péctore, nuestra metáfora iconográfica (Fig. 1), revela la comprensión de la disciplina alcanzada en este segundo epígrafe resaltando la capacidad gnoseológica y creativa de la metáfora que revela el poder normalizador de la disciplina.

\section{Tercer epígrafe: disciplina para el conocimiento escolar, descifrando su dispositivo normalizador}

En la metáfora iconográfica el globo terráqueo puede imaginarse como una proyección del regazo de la maestra en su condición de guardiana del conocimiento escolar. La esfera expresa la función normalizadora de la disciplina y la magnitud de su orden escolar. La maquinaria escolar crea, mediante una alquimia, un artefacto sui generis, la disciplina escolar (Popkewitz, 1994); metáfora que indica la cuantía del proceso normalizador en el conocimiento escolar en tres órdenes imbricados. Primero, orden lógico-analítico, que configura la imagen de un mundo: racional, armonioso, lineal, ahistórico y desvinculado de lo social, extraviándose las implicancias socio-éticas y políticas del proceso por el que la organización del conocimiento está relacionada con la organización del yo. Segundo, orden moral, mediante reglas que determinan la inclusión y exclusión de determinados saberes, así como, sus criterios de normalidad y permisividad. Tercero, orden del poder, mediante la imposición de determinados patrones de conocimiento, ligados al poder como soberanía y como producción, referido a la macro-política de las estructuras históricas, mediante las cuales se distribuye desigualmente el poder, y a la micro-política, donde se construyen las subjetividades (Popkewitz, 1994).

Los órdenes precedentes, muestran que la escolarización es una forma de regulación social donde se configura una imagen de la escuela y del mundo (Popkewitz, 1994). En ese contexto, se inquiere ¿Cuál es el estatuto del saber pedagógico? (Varela, 1995). Este estatuto, se configura mediante dos procesos imbricados: la pedagogización de 
los conocimientos y el disciplinamiento interno de los saberes. Pedagogizar el conocimiento, es impregnar con la norma a saberes y conductas, proceso ligado al nuevo estatuto de la infancia que diferencia entre el mundo del niño y el del adulto y requiere nuevas formas e instituciones educativas, tarea asumida en los países católicos por las órdenes religiosas, por ejemplo, los jesuitas (Varela, 1995). Así, la disciplinarización del conocimiento incluye la transformación de los maestros en los únicos que detentan el saber, unido a la expoliación de un conjunto de prerrogativas a los estudiantes que se transforman, de estudiantes dotados de cierta autonomía, en alumnos o colegiales (Varela, 1995). Unido a ello, se instituye un aparato de penalización y moralización de los colegiales que asocia la adquisición de la verdad y la virtud, a la ascesis y renuncia de uno mismo (Varela, 1995).

En el disciplinamiento interno de los saberes, el epígrafe destaca la scientia, en sus estadios de episteme y scientia, según se trate de un saber contemplativo, dominio de la metafísica, o de un saber medido, dominio de la filosofía natural. ¿Qué scientia? nos sitúa en la naturaleza y su orden científico en cuyo centro está la medición, asevera Galileo; ese orden es causal y está regido leyes naturales, matemáticas, que se reducen a la geometría, según Descartes; posee la estructura de una máquina y un determinismo riguroso, sostiene Newton. Estas claves forman el utillaje que dispone la scientia para la normalización de la institución escolar y su currículum: orden natural, orden causal, determinismo, mecanicismo y medición, subsumidos en la matriz de orden (Oliva 2012). Al examinar la arquitectura de la scientia y su función normalizadora destaca el cambio en las disposiciones fundamentales de la episteme de la cultura occidental donde la comparación es su viga maestra y el orden y la medida, sus figuras fundamentales donde todos los problemas de medida pueden reducirse a los de orden. Luego, la medición es un instrumento principal de la racionalidad disciplinaria, desempeña una función normalizadora y de imposición de un régimen de verdad (Foucault, 1999).

Volvamos, pues, a nuestra fuente iconográfica (Fig. 1), el globo terráqueo preside la relación enseñanza-aprendizaje reafirmando el puesto central del conocimiento escolar en el currículum; tras esta nueva indagación en la disciplina puede ser intitulada Disciplina a fortiori.

\section{Cuarto epígrafe: efecto disciplina en las Bases Curriculares Nacionales}

El conocimiento escolar expresa la dimensión política del currículum; su discurso oficial organiza la percepción y la experiencia siendo capaz de crear sistemas de órdenes dominantes (Popkewitz, 1994). En consecuencia, las Bases Curriculares Nacionales expresan una normalización en el límite, es el efecto disciplina, cuya circunstancia remite a la política educativa chilena 1965 y 2016. 
El período señalado, posee como punto de inflexión la fractura de la democracia chilena que inaugura la dictadura de Pinochet, en un tiempo de sistemática violación de los derechos humanos. El régimen de facto enarbola la idea de subsidiariedad: el hombre posee derechos de origen divino, anteriores y superiores al Estado fundado en el jusnaturalismo de inspiración cristiana (Gobierno de Chile, 1974). Se establece un rol subsidiario del Estado que debe asumir directamente solo aquellas funciones que las sociedades intermedias -formas de asociación situadas entre el individuo y el Estado- no estén en condiciones de cumplir adecuadamente, porque: desborda sus posibilidades (defensa nacional, policía, relaciones exteriores); o su importancia no aconseja dejar entregadas a grupos particulares (empresas estratégica); o envuelven una coordinación general propia del Estado (Gobierno de Chile, 1974). En la escolarización, por ejemplo, participan la familia, la iglesia y el Estado, sin embargo, la familia es el principal agente educativo y sólo cuando no pueda cumplir esta función, el Estado suple el defecto y lo remedia. La transformación en el sistema de financiamiento, de un modelo que subsidia la oferta a otro que subsidia la demanda (voucher), genera un cuasi mercado de la educación; la municipalización traspasa los establecimientos educacionales públicos, desde el Ministerio de Educación a las municipalidades, no obstante, la tuición sobre el currículum y el control de la asistencia de los alumnos para calcular el subsidio, queda bajo potestad del Ministerio de Educación (Oliva, 2017). Consecuencia de ello, profesores y profesoras pierden su condición de funcionarios públicos (Quaresma \& Villalobos, 2016). En el tiempo de estas mudanzas, está vigente en Chile un currículum técnico-instrumental (pedagogía por objetivos) introducido en la reforma educacional de 1965, vinculada a la teoría del capital humano y a la Alianza para el Progreso, modelo curricular que la dictadura radicaliza. Ello da cuenta de un giro de la política educativa hacia la medición del rendimiento escolar que desde el 2001 aparece regulada en estándares (Ministerio de Educación [MINEDUC], 2001); tendencia inscrita en la Reforma Basada en Estándares y regulada en la actual Ley General de Educación (MINEDUC, 2009; Casassus, 2010).

Tras una movilización estudiantil en 2006, que culmina en una crítica estructural a la política educativa, se deroga la Ley Orgánica Constitucional de Enseñanza y promulga la Ley General de Educación, no obstante, mantenerse la Constitución Política sancionada, fraudulentamente, en la dictadura y que todavía está vigente en Chile. La Ley General de Educación crea el Sistema Nacional de Aseguramiento de la Calidad, erigido en dos pilares: una Agencia de Calidad, que vela por la calidad de la educación, y una Superintendencia de Educación, encargada del control y regulación de los recursos fiscales. Se definen estándares de aprendizaje para ordenar a los establecimientos, según el logro de aprendizajes y el cumplimiento de esos estándares y sus respectivas Bases Curriculares Nacionales (MINEDUC, 2009). Estas Bases son el instrumento principal de un currículum centrado en el aprendizaje; su eje curricular es el objetivo de 
aprendizaje o estándar de contenido, que define los aprendizajes y logros terminales esperables para una asignatura al final de cada año escolar y contempla: habilidades, actitudes y conocimientos que buscan favorecer el desarrollo integral de los alumnos y objetivos de aprendizaje transversales para el ciclo, referidos al desarrollo personal y la conducta moral y social de los estudiantes (MINEDUC, 2013). La estandarización del conocimiento aparece unida a un sistema de medición también estandarizado, el Sistema Nacional de Medición de la Calidad de la Educación (SIMCE), que pone en línea los estándares de contenidos con los estándares de desempeño.

La Reforma Basada en Estándares es impulsada por las elites políticas, empresariales, tecno-burocráticas y mediáticas interesadas en mantener el vínculo entre economía y educación, quienes sostienen que la medición externa y objetiva de resultados, referidos a estándares (tipo, modelo, patrón), es eficaz para mejorar la calidad y equidad de este modelo, sin embargo, se trata de un mecanismo de medición de origen conductista, unido a la rendición de cuentas (accountability) y que busca aumentar el control externo sobre el proceso de aprendizaje (Casassus, 2010). En este contexto, el cuarto epígrafe argumenta que el disciplinamiento del conocimiento escolar en las Bases Curriculares chilenas vigentes, permite observar el papel de la disciplina en la estandarización del currículum técnico expresada en el binomio racionalidad disciplinaria/racionalidad técnicoinstrumental. La racionalidad disciplinaria, está representada por el proceso normalizador que desempeña el estándar, como dispositivo principal, y su concreción en 3 tipos de estándares: de aprendizaje, de contenido y de medición. Su acoplamiento en torno a un eje, el objetivo de aprendizaje, configura una matriz disciplinaria, que es parte de un orden y genera un orden, cuyos efectos sobre el conocimiento escolar son: homogeneizar, simplificar, alinear, controlar, mecanizar, en suma, normalizar. La cercanía entre las acciones de alinear y alienar, permiten inferir las consecuencias del poder normalizador de la disciplina; estrategia de normalización en cuyo trasluz es posible descubrir los procesos de pedagogización de los conocimientos y su disciplinamiento interno que configuran el estatuto del saber pedagógico (Varela, 1995). La scientia y su medición, es el fundamento del currículum técnico-instrumental donde se aloja la lógica de los estándares fundados en la medición de resultados. Nótese el orden mecanicista y determinista de la matriz Bases Curriculares Nacionales, que regula un aprendizaje o desempeño observable: definido conceptual y operacionalmente mediante criterios e indicadores. El mentado objetivo requiere de una disección y control de la eficiencia de su trayectoria de aprendizaje: punto de inicio (formulación del objetivo); desarrollo (actividades y medios) y punto de llegada (estándares de medición), incorporando la perspectiva medios-fin propia del currículum técnico-instrumental.

Imaginemos disciplina in péctore hoy: alumnas y maestra en cualquier aula chilena reguladas por las Bases Curriculares, su código curricular subyacente permite inferir la funcionalidad del currículum técnico-instrumental al orden neoliberal en los objetivos 
fijados en consonancia con los mercados económicos, según su postura eficientista erigida en el binomio estándar/medición, que estratifica a los alumnos de acuerdo a su origen socioeconómico y los puntajes obtenidos en pruebas estandarizadas diezmando, con ello, las posibilidades de movilidad social.

Disciplina ad maximis ad mínima es el título que este cuarto epígrafe permite crear para la metáfora iconográfica (Fig.1), al descubrir el efecto disciplina en las Bases Curriculares Nacionales: establece el límite y los andamios de la arquitectura de la desigualdad y fragmentación social en su funcionalidad con la reproducción social.

\section{Quinto epígrafe: encrucijada de la disciplina escolar e (im)posibilidad de la educación}

Disciplina virtuosa y disciplina viciosa, en vis a vis, son una apertura para pensar las posibilidades y límites de la educación. La disciplina es el espíritu del orden que hace a la educación, así lo evidencian autores situados en diferentes perspectivas para quienes la disciplina proporciona las normas -máximas morales- para disponer al ser humano a desarrollar su humanidad y participar ordenadamente en la vida social. El vínculo entre disciplina y educación aparece inscrito en el imperio del orden, presente en el nacimiento del discurso pedagógico moderno (Beltrán, 2000). Allí el orden es el alma de las cosas, aquello que les permite llegar a ser y mantenerse en su modo de ser; el cosmos u orden de la Naturaleza, constituye el supremo orden al cual deben sujetarse todos los órdenes particulares, ello es lo que permite que cada una de las cosas y criaturas permanezcan dentro de sus límites, según el mandato de la Naturaleza (Comenius, 2000). En ese orden, la disciplina constituye un modo cierto, en virtud del cual los discípulos se hacen verdaderamente discípulos, permitiendo la corrección de las costumbres en aquellos que se aparten del recto camino, antes que, siendo usada con motivo de los estudios y las letras (Comenius, 2000). Para Kant (1921), el orden de la vida supone un orden que le precede, el orden de la educación, el espíritu de este orden es la obediencia, vale decir, la disciplina que proporciona la educación. Esto exige cultivar, tanto en el individuo como en la sociedad, un conjunto de disposiciones, v. gr., habilidades y rasgos del carácter, para que el hombre pueda vivir como un ser libre. La disciplina constituye la finalidad de la educación, debe preparar el camino para el desarrollo de buenos hábitos, previniendo la formación de aquellos considerados perniciosos; la disciplina permite transitar del estado de animalidad, al estado de humanidad (Kant, 1921). En esta misma línea, Herbart (1806), plantea que el gobierno de los niños constituye el fin de la educación en general, este gobierno "no tiene que alcanzar ningún fin en el espíritu del niño, sino, únicamente, establecer el orden" (p. 91). Pensamiento que distingue entre instrucción y educación, entre la ciencia que se ha de comunicar, y el gobierno de los niños. El gobierno introducido en 
la educación, posee como misión mantener el orden y proporcionar a la pedagogía el principio de una gran ordenación. Este gobierno es necesario, porque el hombre viene al mundo desprovisto de voluntad e incapaz de una voz moral, por ello, es imperativo una educación moral cuya finalidad es que las ideas de justicia y bien, lleguen a ser los objetos de la voluntad, y determinen la esencia de la personalidad (Herbart, 1806). En este contexto, la disciplina es la acción inmediata que se ejerce en el espíritu del niño para educarle; ella, no debe confundirse con el gobierno, pese a que ambas comparten su ámbito de acción, el espíritu. El orden proveedor del gobierno junto a la acción que ejerce la disciplina sobre el espíritu del niño, permite disponerlo hacia la instrucción cuyo objetivo último es la virtud; es éste orden disciplinario el que posibilita los órdenes subsiguientes (Herbart, 1806).

Orden social, disciplina y educación moral aparecen, indisolublemente, unidos para Durkheim (2002), baste advertir que el primer elemento de la moralidad es el espíritu de la disciplina, pues, la moral es esencialmente una disciplina. Su moral, laica y racional, es la viga maestra para la construcción del orden social que debe transmitir el sistema escolar y se articula en tres elementos: el espíritu de la disciplina, la vinculación a los grupos sociales y la autonomía de la voluntad. La disciplina, es la que da unidad a estos elementos, por ello constituye el primer elemento de la moralidad que es, esencialmente, una disciplina encaminada al deber y al bien.

Este halo de bondad de la disciplina queda desdibujado al observar la disciplina en la mentada época clásica abordada en el segundo epígrafe. Parece posible pensar que el reto político que plantea la mentada encrucijada de la disciplina, sea el fortalecimiento del espacio público y la promoción de un proyecto político erigido en la racionalidad, primer fundamento de la dignidad humana. Su búsqueda es la democracia, cuya esencia es el riesgo, la aventura y ese carácter pertenece a su propia naturaleza y se funde en un desafío a la vida humana (Millas, 1974), precisamente, aquí radica la (im)posibilidad de la educación.

Disciplina, ab exordio vitae, se intitula la fuente iconográfica al calor de las vicisitudes de la disciplina en la encrucijada de lo escolar (Fig. 1).

\section{Sexto epígrafe: exhortación a una Política de la Reparación. Conclusión}

¿Podríamos pensar en una Política de Reparación frente a la vulneración del derecho a la educación en Chile? Repárese en la disciplina, estatuto matriz de lo escolar, engendra el orden y ordena. Su expresión en las Bases Curriculares Nacionales ilustra esta vocación normalizadora. Por ello, pensar en términos de dispositivos permite desnudar la trama de la vida escolar, así se expresa en la retórica discursiva que acompaña su promulgación; el saber que se manifiesta en sus formas de enunciación y visibilidad al observar el continuo entre las Bases Curriculares Nacionales y sus antecesores: 
el Marco Curricular Nacional y la pedagogía por objetivos. Allí, la formación histórica del objetivo educacional, considerado como dispositivo del currículum instrumental enseña, en su fondo de permanencia, la trayectoria de sus mudanzas a: aprendizaje esperado, en la Ley Orgánica Constitucional de Enseñanza y objetivo de aprendizaje en la Ley General de Educación. Las relaciones de fuerza atraviesan todo lo anterior, baste observar el impacto del binomio estandarización/medición en el aprendizaje y contenidos, según la lógica de resultados de aprendizaje en la consagración de la reproducción por la vía de la desigualdad y la fragmentación social. Repárese, también, en el efecto disciplina en el currículum nacional, la normalización del conocimiento escolar en la lógica de la estandarización está implicada en la construcción del orden neoliberal; su noción de calidad subyacente, define las prioridades para la educación según un análisis económico, junto a la determinación y medición de estándares acorde a las regulaciones externas (Oliva y Gascón, 2016). En suma, racionalidad disciplinaria y construcción de la desigualdad constituyen una manifestación del círculo vicioso de la disciplina. La encrucijada de la disciplina es, así, el lugar donde germina la Política de Reparación como un proyecto perpetuo de emancipación política para fundar un nuevo orden, tal como lo ilustra para el proyecto pedagógico, Francisco Beltrán (2000),

un proyecto perpetuo de emancipación política; es un proyecto de lucha permanente para fundar un nuevo orden, a sabiendas de que, contenida en el mismo, debe estar la semilla de su propia transformación. El papel que en ese proyecto le corresponde a la disciplina es el de trazar la retícula en el cual se inscribe nuestro presente y, en consecuencia, señala las posibilidades de caminar, aun lastrados por la dignidad siempre maltrecha del obediente, hacia un paisaje de libertad (p. 73).

Volvamos, pues, a nuestra fuente iconográfica (Fig.1) inspirados en el señalado proyecto de emancipación política, el devenir de la investigación ha permitido intitularla, sucesivamente: Disciplina in péctore; Disciplina a fortiori; Disciplina ab maximus ad minima, en fin, Disciplina, ab exordio vitae, epígrafes todos los cuales permiten exhortar a la Reparación (Oliva, 2016). La ambivalencia misma del concepto reparar traza la retícula para erigir la matriz de este nuevo orden fundado en lo mejor del ayer: la Política de la Reparación. En efecto, reparar es enmendar y también es darse cuenta; una simple epojé enseña que reparar es desagraviar, reanimar, alentar, vigorizar, insuflar valor, enmendar, en fin, remediar. También, es darse cuenta, advertir, tener o tomar en cuenta, atender, considerar, percibir, en fin, despertar a una vigilia alerta (Larousse, 2014). Reparar es instituir un nuevo orden, fundado en la dignidad humana, para transmutar aquél que se desmarca de la virtud, arquitectura de un sueño político articulado en la mejor tradición pedagógica, la educación democrática -que si bien participa de 
esa tradición merece ser subrayada- y el derecho a la educación en la tradición de los derechos humanos (Estêvão, 2011). Su afán es impulsar la trasmutación ética y política del círculo vicioso de la disciplina, a su espiral virtuosa, rito fundacional donde la virtud de la dignidad cimente todos los derechos y la democracia; sueño político donde germina nuestra Política de Reparación.

Obs: Artigo dedicado a Patricio Sanhueza Vivanco, Político Principal

Nota

1 El artículo se sustenta en la investigación doctoral realizada en la Universidad de Valencia, El tiempo un modo de orden. Una paradoja en la historia como disciplina escolar (Oliva, 2012). Tras la dictadura chilena, mis profesores José Gimeno Sacristán, Francisco Beltrán LLavador y Jaume Martínez Bonafé, me revelaron las claves para la búsqueda del tiempo perdido y la resignificación del tiempo ganado en el exilio interior chileno, sus enseñanzas han cristalizado en la Política de la Reparación expuesta en este trabajo.

\section{Referencias}

Agamben, G. (2011) ¿Qué es un dispositivo? Sociológica, año 26(73), 249-264.

Beltrán, F. (2000). Hacer pública la escuela. Santiago de Chile: LOM ediciones.

Casassus, J. (2010). Las reformas basadas en estándares: un camino equivocado. Educere et educare. Revista de Educação, 5(9), 85-107. Disponível em: http://erevista.unioeste.br/index.php/ educereeteducare/article/view/4082

Comenius, J.A. (2000).Didáctica Magna. México D.F.: Porrúa (1ª ed.1633-1638).

Disciplina in péctore. Maestra y alumnas. Escuela de Recoleta Santiago de Chile. Mitad inferior, ellas mismas volteadas en $180^{\circ}$. Adaptación autora de fotografía Colección fotográfica del Museo de la Educación Gabriela Mistral de Santiago de Chile. Recuperado de http: //www.memoriachilena. cl/602/w3-article-74370.html

Durkheim, E. (2002). La educación moral. Madrid: Morata.

Estêvão, C. (2011). Democracia, Direitos Humanos e Educação. Para uma perspectiva crítica de educação para os direitos humanos. Revista Lusófona de Educação, 17, 11-30. Disponível em http://revistas.ulusofona.pt/index.php/rleducacao/article/view/2361, 2018-07-01.

Freire, P. (1993). Professora sim tia não. A quem ousa ensinar. São Paulo: Olho d’Água.

Foucault, M.(2014). Vigiar e punir: nascimento de prisão. Petrópolis, RJ: Vozes

Foucault, M. (1999): Las palabras y las cosas. México D.F.: Siglo XXI.

Gobierno de Chile. (1974). Declaración de Principios del Gobierno de Chile. Santiago de Chile: Gabriela Mistral.

González Ramella, A. I. (2003). Poder y discursos en la construcción social de las identidades docentes universitarias. Tesis de doctorado no publicada, Universidad de Valencia, Valencia, España. 
Herbart, J. F. (1806). Pedagogía general derivada del fin de la educación. Madrid: Ediciones de La Lectura.

Kant, E. (1981).Traité de pédagogie, Paris: Hachette.

Marías, J. (1971). Historia de la filosofía. Madrid: Revista de Occidente.

Millas, J. (1974). De la tarea intelectual. Santiago de Chile: Universitaria.

Ministerio de Educación (2016). Ley No 20.903 Crea el sistema de desarrollo profesional docente y modifica otras normas. Santiago de Chile, Ministerio de Educación, 2016. Recuperado de http:// ben.cl/1uzzn.

Ministerio de Educación (2013). Síntesis Nuevas bases Curriculares. Santiago de Chile: MINEDUC.

Ministerio de Educación (2009). Ley № 20.370. Establece la Ley General de Educación LGE. Santiago de Chile. Biblioteca del Congreso Nacional de Chile. Recuperado de http://www.ley.chile.cl

Ministerio de Educación (2001). Estándares de desempeño para la formación inicial de docentes. Santiago de Chile, Ministerio de Educación. Recuperado de http://ww2.educarchile.cl/Us2erFiles/ P0001/File/estandares_formacion_docentes.pdf

Organisation for Economic Co-operation and Development. (2018). Economic Surveys Chile February 2018 OVERVIEW Economic and Development Review Committee of the OECD. Retrieved from http://www.oecd.org/eco/surveys/Chile-2018-OECD-economic-survey-overview.pdf (Cita traducida por la autora del artículo).

Oliva, M. A. (2017). Arquitectura de la política educativa chilena (1990-2014): el currículum, lugar de la metáfora. Revista Brasileira de Educação, 22 (69), 405-428. Disponível em https://dx.doi. org/10.1590/s1413-24782017226921

Oliva, M. A. (2016).Grito por el Derecho a la Educación en Chile. Marcha de los paraguas y movilización estudiantil de 2011. Altre modernità. Milano: Universita degli Studi di Milano. Especiale. Nuevos movimientos sociales, política y derecho a la educación. Disponibile in https://riviste.unimi.it/index. php/AMonline/article/viewFile/7105/6915

Oliva, M. A. (2012). El tiempo un modo de orden. Una paradoja en la historia como disciplina escolar. Tesis de doctorado no publicada. Valencia: Universidad de Valencia. Recuperado de https://core. ac.uk/download/pdf/71005679.pdf

Oliva, M. A. y Gascón i Martín, F. (2016). Estandarización y Racionalidad Política Neoliberal: Bases Curriculares De Chile. Cadernos CEDES, 36 (100), 301-318. Disponível em https://dx.doi. org/10.1590/cc0101-32622016171349

Popkewitz, T. (1994). Política, conocimiento y poder: algunas cuestiones para el estudio de las reformas educativas. Revista de Educación, (305), 103-137. 
Quaresma, M.L. \& Villalobos, C. (2016). Mercantilização, competitividade e accountability no sistema de ensino em Portugal: o que se pode aprender da experiência chilena. Revista Lusófona de Educação, 32, 31-47 Disponível em http://revistas.ulusofona.pt/index.php/rleducacao/article/view/5507, consultado en 2018-07-01.

Varela, J. (1995). El estatuto del saber pedagógico. In V.V.A.A.: Volver a pensar la educación. (61-69). Madrid: Morata.

María Angélica Oliva Investigadora Centro de Estudios Avanzados y Profesora Postgrado

Universidad de Playa, Chile.

Email: angelica.oliva@upla.cl Orcid: https://orcid.org/0000-0002-2779-2517

Correspondência

María Angélica Oliva Ureta Centro de Estudios Avanzados (CEA) Universidad de Playa Ancha Traslaviña 450, Viña del Mar, Chile

Data de submissão: Janeiro de 2018 Data de avaliação: Março de 2018 Data de publicação: Abril 2019 